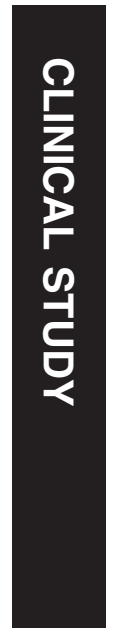

The Medical Department SIFI SpA

Catania, Italy

Correspondence:

$\checkmark$ Papa, MD PhD

Medical Department

SIFI SpA

Via Ercole Patti 36

95020 Lavinaio

Catania, Italy

Tel: 390957922375

Fax: 390957893451

E-mail: vincenzo_papa@ sifi.it

\section{Topical naproxen sodium for inhibition of miosis during cataract surgery. Prospective, randomized clinical trials}

V Papa, S Russo, P Russo, A Di Bella, M Santocono, G Milazzo and Naproxen Study Group

\begin{abstract}
Purpose To assess corneal penetration of naproxen sodium and its efficacy in maintaining intraoperative mydriasis during cataract surgery.

Methods Two double blind studies have been performed comparing the efficacy of naproxen ophthalmic solution to that of placebo or diclofenac in inhibiting preoperative miosis. Study No. 1 was a placebocontrolled study and involved 194 patients undergoing extracapsular cataract extraction. Study No. 2 was an active-controlled study (vs diclofenac) concerning 214 patients undergoing phacoemulsification. In both studies treatment started the day before surgery. A balanced salt solution containing adrenaline was used in all patients. Pupil size was measured prior to the corneal section and at the end of surgery. An aqueous humor sample was taken immediately before corneal incision in a subset of 20 patients to measure naproxen aqueous concentration.

Results In both studies the pupillary diameter decreased during surgery within each treatment group in a statistically significant manner $(P<0.001)$. Naproxen was more effective than placebo $(P<0.01)$ and as effective as diclofenac in controlling pupil diameter regression during cataract. Mean concentration level of naproxen in the aqueous humor was $372.3 \mathrm{ng} / \mathrm{ml}$.

Conclusions Naproxen sodium ophthalmic solution penetrates the cornea and it is effective in maintaining intraoperative mydriasis.

Eye (2002) 16, 292-296. DOI: 10.1038/

sj/eye/6700124
\end{abstract}

Keywords: aqueous humor; cataract surgery; NSAID; naproxen; mydriasis

Introduction

The maintenance of mydriasis is important during cataract surgery to facilitate uncomplicated cortex removal and intraocular lens insertion. ${ }^{1}$ Preoperative adequate pupil dilatation is generally achieved using topical adrenergic agonists, such as phenylephrine in combination with a cholinergic antagonist such as cyclopentolate. Usually irrigating solutions of adrenaline are also used intracamerally to help maintain mydriasis during surgery. Nevertheless, in many eyes subsequent onset of miosis begins soon after the anterior chamber is entered. This reaction is thought to be caused by the effect on the iris sphincter (constriction) of prostaglandins (PGs) and other mediators that are released when blood-aqueous barrier breakdown occurs during surgery. ${ }^{2}$ Nonsteroidal antiinflammatory drugs (NSAIDs) are powerful inhibitors of PG synthesis and given topically are effective in decreasing the rate of miosis. ${ }^{3,4}$

Naproxen sodium is a well-known NSAID with established anti-inflammatory activity. ${ }^{5}$ As an ophthalmic solution, it is able to decrease aqueous levels of proteins, polymorphonuclear leukocytes and $\mathrm{PGE}_{2}$ following experimentally induced inflammation in rabbits. ${ }^{6,7}$ In addition, topical sodium naproxen successfully maintains mydriasis induced by atropine following paracentesis in the rabbit eye. ${ }^{7}$ Safety and tolerance of $0.2 \%$ naproxen ophthalmic solution have been shown in healthy volunteers. ${ }^{8}$ This ophthalmic solution is also 
effective in preventing and controlling ocular

inflammation after cataract surgery. ${ }^{9}$

In the present study we assessed the ability of naproxen eye drops to penetrate the cornea and to maintain mydriasis during the two most common cataract operation procedures, ie extracapsular cataract surgery and phacoemulsification.

\section{Patients and methods}

The effect of naproxen ophthalmic solution on the maintenance of intraoperative mydriasis was analysed in two separate studies.

Study No. 1 was a randomized, double-blind, parallel group, placebo-controlled study conducted in patients who were to undergo extracapsular cataract surgery. In this study the placebo used was the naproxen vehicle.

Study No. 2 was a randomized, double-blind, parallel group, active-controlled study conducted in patients selected to undergo cataract extraction by phacoemulsification. Diclofenac was chosen as the active control in this study because it is considered worldwide the 'gold standard' in the NSAID group.

In both studies patients of either sex, at least 40 years old and scheduled to undergo cataract surgery were enrolled. Exclusion criteria for both studies covered any ocular pathology (including diabetic retinopathy), herpes infection, ocular medications other than topical beta-blockers or artificial tears, other ocular surgery or laser treatment in the eye to be operated within 6 months preceding surgery, known or suspected allergy to any of the ingredients in the study medications and use of topical or systemic steroids or NSAIDs in the 15 days before surgery.

Surgeries were performed in several centers located in Italy (see Appendix) using standard procedures, ie corneal section under a local or topical anaesthetic. Intraocular lidocaine was not employed during surgery. A balanced salt solution containing adrenaline (1 part per million) was used to irrigate the anterior chamber.

Local ethics committees approved study protocols. All patients gave written informed consent. Medical commitment texts, eg Declaration of Helsinki, GCP etc were observed.

In each study, patients were assigned using a computer-generated randomization list into two treatment groups. All patients received routine preoperative dilating drops consisting of $1 \%$ cyclopentolate, $1 \%$ tropicamide and $2.5 \%$ phenylephrine starting $1 \mathrm{~h}$ before surgery.

The treatment with the study medications (ie naproxen or placebo in Study No. 1; naproxen or diclofenac in Study No. 2) started the day before surgery (one drop t.i.d. in the conjunctival sac of the affected eye). On the day of surgery, treatment was administered every half-hour during the 2-h period prior to surgery (four doses).

Pupillary diameter was recorded in millimeters by the surgeon using a caliper prior to the corneal section and at the end of surgery. Pupil diameter and other numerical data were analyzed by Student's $t$-test. Categorical data were analyzed with frequency tables and treatments were compared with the chi-square $\left(\chi^{2}\right)$ method. The 0.05 (two-tailed test) was chosen as the level of significance.

In a subset of 20 consecutive patients enrolled in Study No. 2, an aqueous humor withdrawal was performed after obtaining a fully informed consent. At the end of the study, the blind randomization code was opened and naproxen sodium was measured in the aqueous humor in the subgroup of 10 patients treated with naproxen. The last drop of topical NSAID was administered $30 \mathrm{~min}$ prior to obtaining the aqueous fluid sample. From each patient, $0.1 \mathrm{ml}$ of aqueous fluid was withdrawn at the start of the surgical procedure using a standard insulin syringe. Each sample was placed in appropriately labeled tubes and frozen immediately at $-20^{\circ} \mathrm{C}$. Each sample was then analyzed in one batch for naproxen content through a sensitive and specific high-performance liquid chromatography (HPLC) validated method. The lower limit of quantitation of this assay was $50 \mathrm{ng} / \mathrm{ml}$.

\section{Results}

A total of 408 patients were randomized in the two studies; of these, 203 were treated with naproxen.

One hundred and ninety-four patients were enrolled in Study No. 1 and underwent cataract surgery performed by extracapsular extraction: of these, 97 were treated with naproxen and 97 with placebo.

Two hundred and fourteen patients were selected in Study No. 2 and underwent cataract extraction by phacoemulsification: 106 were treated with naproxen and 108 with diclofenac.

In both studies no statistically significant differences in the demographic data were noted between groups of treatment. All enrolled patients were Caucasian. The subjects' age ranged from 41 to 91 years (Table 1).

\section{Study No. 1 (Placebo-controlled)}

Summary of data regarding the effect of naproxen or placebo on pupil size is shown in Table 2. There were no statistically significant differences between treatment groups for the pupillary diameter at the beginning of surgery ( $t$-test, $P>0.5)$. On the contrary, 
Table 1 Age of patients

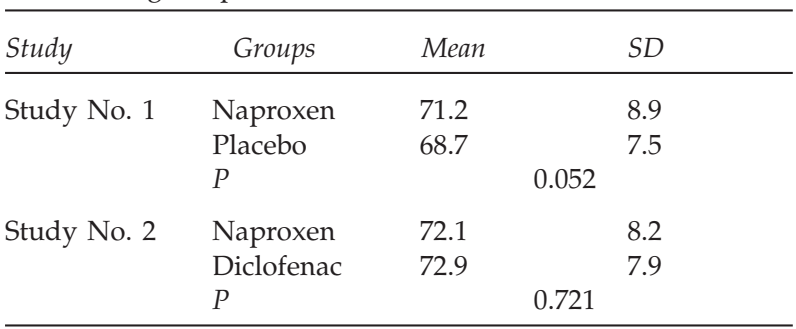

a statistically significant difference was observed at the end of surgery ( $t$-test, $P<0.01$ ). Accordingly, for the diameter difference (end minus beginning), a statistically significant difference in favor of naproxen was detected ( $t$-test, $P<0.01$ ). A decrease of pupil diameter after surgery $>2 \mathrm{~mm}$ compared with the preoperative value was observed in more than $75 \%$ of patients in the placebo group (73/97) and in only $39 \%$ of patients in the naproxen group (38/97). This difference was highly statistically significant ( $\chi^{2}$ test, $P$ $<0.001$ ). Within each treatment group, the pupillary diameter decreased in a statistically significant manner (paired $t$-test, $P<0.001$ ).

\section{Study No. 2 (Active-controlled)}

The results of the study comparing the effect of naproxen to that of diclofenac on pupil size are shown in Table 3. There were no statistically significant differences between treatment groups for the pupillary diameter both at the beginning and end of surgery. Accordingly, the diameter difference was not statistically significant between the two groups. As observed in the study with placebo, the decrease of the pupillary diameter within each treatment group was statistically significant (paired $t$-test, $P<0.001$ ).

\section{Naproxen sodium in aqueous humor}

In 20 consecutive patients enrolled in Study No. 2, an aqueous humor withdrawal was performed. At the end of the study, the blind randomization code was opened and naproxen sodium was measured in the aqueous humor of those patients who were treated with naproxen $(n=10)$. The HPLC analysis indicated that the mean concentration level of active principle in the aqueous humor reached $372.3 \pm 189.0 \mathrm{ng} / \mathrm{ml}(1.5 \pm 0.7$ $\mu \mathrm{M})(\mathrm{m} \pm \mathrm{SD})$.

\section{Discussion}

Activation of phospholipase $\mathrm{A}_{2}$ after tissue injury breaks down cell membrane phospholipids to arachidonic acid. This is then converted either into PGs by cyclo-oxygenase (COX) or into hydroxy acids and leukotrienes by 5-lipoxygenase. PGs cause miosis, increase in vascular permeability of the blood-ocular barriers and changes in IOP. ${ }^{2,3}$ NSAIDs decrease PG

Table 2 Study No. 1: Effect of naproxen and placebo ophthalmic solutions on pupillary diameter

\begin{tabular}{|c|c|c|c|}
\hline & Naproxen & Placebo & \\
\hline \multirow[t]{2}{*}{ No. of patients } & 97 & 97 & \\
\hline & & & $\begin{array}{c}P \text { value (between } \\
\text { treatment) }\end{array}$ \\
\hline Pupillary diameter at the beginning of surgery $(\mathrm{mm})($ mean $\pm S D)$ & $7.99 \pm 0.89$ & $8.07 \pm 1.05$ & 0.552 \\
\hline Pupillary diameter at the end of surgery $(\mathrm{mm})($ mean $\pm \mathrm{SD})$ & $5.78 \pm 1.27$ & $5.02 \pm 1.21$ & $<0.01$ \\
\hline Mean diameter difference (end minus beginning) $(\mathrm{mm})$ & -2.21 & -3.05 & $<0.01$ \\
\hline$P$ value (within treatment) & $<0.001$ & $<0.001$ & \\
\hline
\end{tabular}

Table 3 Study No. 2: Effect of naproxen and diclofenac ophthalmic solutions on pupillary diameter

\begin{tabular}{|c|c|c|c|}
\hline & Naproxen & Diclofenac & \\
\hline \multirow[t]{2}{*}{ No. of patients } & 106 & 108 & \\
\hline & & & $\begin{array}{c}P \text { value (between } \\
\text { treatment) }\end{array}$ \\
\hline Pupillary diameter at the beginning of surgery $(\mathrm{mm})($ mean $\pm \mathrm{SD})$ & $7.53 \pm 1.27$ & $7.44 \pm 1.31$ & 0.777 \\
\hline Pupillary diameter at the end of surgery $(\mathrm{mm})($ mean $\pm \mathrm{SD})$ & $6.69 \pm 1.57$ & $6.84 \pm 1.62$ & 0.331 \\
\hline Mean diameter difference (end minus beginning) $(\mathrm{mm})$ & -0.65 & -0.60 & 0.077 \\
\hline$P$ value (within treatment) & $<0.001$ & $<0.001$ & \\
\hline
\end{tabular}


production by inhibiting COX activity on arachidonic acid. ${ }^{3,4}$

It has been well known for many years that systemically given naproxen is able to reduce ocular inflammation. ${ }^{10-13}$ In addition it has been shown that the topical formulation of sodium naproxen used in the present study is able to reduce both clinical and biochemical signs of ocular inflammation in different experimental animal models. ${ }^{6,7}$ Furthermore, naproxen has been shown to be effective in maintaining mydriasis in a rabbit model. ${ }^{7}$ The global safety and local tolerance of naproxen eye drops have also been tested in healthy volunteers and found acceptable after either single or repeated conjunctival administrations. ${ }^{8}$ A recent study has also shown that this new ophthalmic solution is as effective as diclofenac sodium in controlling postsurgical inflammation in patients who undergo cataract extraction. ${ }^{9}$

Cataract extraction is associated with increased PG levels in the aqueous. ${ }^{2}$ These PGs are released as part of the inflammatory response and may enhance the action of the iris sphincter causing pupil constriction before and during surgery. Topical NSAIDs are able to reduce miosis by inhibiting PG generation in the aqueous. ${ }^{3,4,14-18}$ Aqueous humor sampling is at present the best method to study ocular availability of such drugs during surgery. Accordingly, flurbiprofen, indomethacin and diclofenac penetrate the cornea when administered preoperatively in patients having cataract extraction, reaching concentrations in the range of 30-200 ng/ml. ${ }^{14,19}$ Our study suggests that topical naproxen is also able to constrain aqueous PG production. In fact the naproxen concentration gained in human aqueous (approximately $370 \mathrm{ng} / \mathrm{ml}=1.5$ $\mu \mathrm{M})$ is able to inhibit COX activity by at least $50 \%$ in selected human and animal tissue. ${ }^{5}$

Preoperative good pupil dilatation is generally achieved using a combination of adrenergic agonists and cholinergic antagonists. Usually adrenaline is also used intracamerally as an irrigating solution to help maintain mydriasis during surgery. ${ }^{1}$ NSAIDs are mainly useful as an adjunctive to standard preoperative dilating regimens, but not as sole agents for the inhibition of intraoperative pupillary constriction. Adequate mydriasis is especially required during phacoemulsification for performing capsulorhexis and complete removal of lens cortical remnants.

The two studies reported here differ in the type of surgical procedure used to remove cataract (extracapsular extraction $v$ s phacoemulsification). In both studies naproxen was effective in maintaining intraoperative mydriasis. Intraoperative iris touch and/or iris prolapse may influence the effect of NSAID on pupil dilation. ${ }^{2}$ However, as a possible limitation of the study, such information was not available on our groups of patients.

In the study involving patients who underwent extracapsular extraction (Study No. 1), a pre-surgical pupillary diameter larger than $5 \mathrm{~mm}$-used as a surgical significant cut-off ${ }^{20}$ — was maintained by using adrenaline in the irrigating fluid in the absence of NSAIDs (placebo group). The concept that NSAIDs may play an additional role in inhibiting miosis was confirmed in this series of data. The additive effect of naproxen was limited (approximately $0.8 \mathrm{~mm}$ ) but statistically significant.

These results were also confirmed in the study involving patients who underwent cataract surgery by phacoemulsification (Study No. 2), where naproxen was found as effective as diclofenac. Interestingly, a lower mean pupillary diameter regression was observed in this study, compared with that observed in the study involving extracapsular extraction (approximately 0.6 $\mathrm{mm}$ vs $2.2 \mathrm{~mm}$, respectively). The different extent of surgical trauma between the two techniques may explain these results.

The data included in the present report add to the currently published literature confirming that the standard preoperative dilating therapy and the intraocular irrigation with adrenaline are still the most important factors in preventing surgical miosis. However, combination with a new ophthalmic NSAID containing sodium naproxen produces a greater inhibition of the miotic response during cataract surgery performed by either extracapsular extraction or phacoemulsification.

\section{Acknowledgements}

We thank C Gramolini and J Klinger for the statistical analysis of data.

The study was entirely supported by SIFI SpA. None of the authors has a financial interest in the product.

\section{References}

1 Rowen S. Preoperative and postoperative medications used for cataract surgery. Curr Opin Ophthalmol 1999; 10: 29-35.

2 Neufeld AH, Sears ML. Prostaglandins and eye. Prostaglandins 1973; 4: 157-175.

3 Flach AJ. Cyclo-oxygenase inhibitors in ophthalmology. Surv Ophthalmol 1992; 36: 259-284.

4 Koay P. The emerging roles of topical non steroidal anti inflammatory agents in ophthalmology. Brit J Ophthalmol 1996; 80: 480-485.

5 Todd PA, Clissold SP. Naproxen: a reappraisal of its pharmacology and therapeutic use in rheumatic diseases and pain states. Drugs 1990; 40: 91-137. 
6 Spampinato S, Marino A, Bucolo C, Canossa M, Bachetti $\mathrm{T}$, Mangiafico S. Effects of sodium naproxen eye drops on rabbit ocular inflammation induced by sodium arachidonate. J Ocular Pharmacol 1991; 7: 125-133.

7 Bucolo C, Spadaro A. Effect of sodium naproxen on inflammatory response induced by anterior chamber paracentesis in the rabbit. J Pharm Pharmacol 1995; 47: 708-712.

8 Papa V, Waitzinger J , Pabst G, Milazzo G, Muller M, Marrano $\mathrm{M}$ et al. Safety and tolerability of naproxen ophthalmic solution in comparison to placebo. Int J Clin Pharm Ther 1999; 37: 133-140.

9 Papa V, Milazzo G, Santocono M, Servolle V, Sourdille P, Santiago PY et al. Naproxen ophthalmic solution in the management of inflammation after phacoemulsification. $J$ Cataract Refract Surg 2002; 28: 321-327.

10 Fleisher LN, Ferrel JB, Smith MG, McGahan MC. Lipid mediators of tumor necrosis factor-alpha induced uveitis Invest Ophthalmol Vis Sci 1991; 32: 2393-2399.

11 Nielsen CB. The effect of the prostaglandin-inhibitor naproxen on the endothelial cell-loss after cataract extraction. Acta Ophthalmol 1983; 61: 102-107.

12 Nielsen CB. Prostaglandin inhibition and central corneal thickness after cataract extraction. Acta Ophthalmol 1982; 60: 252-258.

13 Scott JA, Clearkin LG. Surgically induced diffuse scleritis following cataract surgery. Eye 1994; 8: 292-297.

14 Gimbel H, Van Westenbrugge J, Cheetham JK, DeGryse R, Garcia CG. Intraocular availability and pupillary effect of flurbiprofen and indomethacin during cataract surgery. J Cataract Refract Surg 1996; 22: 474-479.

15 Antcliff RJ, Trew DR. The maintenance of per-operative mydriasis in phacoemulsification with topical diclofenac sodium. Eye 1997; 11: 389-391.

16 Salomon KD, Turkalj JW, Whiteside SB, Stewart JA, Apple DJ. Topical 0.5\% ketorolac vs 0.03\% flurbiprofen for inhibition of miosis during cataract surgery. Arch Ophthalmol 1997; 115: 1119-1122.

17 Thaller VT, Kulshrestha MK, Bell K. The effect of preoperative topical flurbiprofen or diclofenac on pupil dilatation. Eye 2000; 4: 642-645.
18 Gimbel HV. The effect of treatment with topical nonsteroidal anti-inflammatory drugs with and without intraoperative epinephrine on the maintenance of mydriasis during cataract surgery. Ophthalmology 1989; 95 $585-588$.

19 Killer HE, Borruat FX, Blumer BK, Herbort CP, Jauch A. Corneal penetration of diclofenac from a fixed combination of diclofenac-gentamicin eyedrops. J Cataract Refract Surg 1998; 24: 1365-1370.

20 Corbett MC, Richards AB. Intraocular adrenaline maintains mydriasis during cataract surgery. $\mathrm{Br} \mathrm{J}$ Ophthalmol 1994; 78: 95-98.

\section{Appendix}

\section{Naproxen Study Group}

\section{Coordinators:}

Aldo Caporossi, Cattedra di Oftalmologia Università di Siena Francesco Ponte, Cattedra di Oftalmologia Università di Palermo

\section{Principle Investigators:}

Giovanni Anselmetti, Divisione di Oculistica Ospedale Maria Vittoria, Torino

Salvatore Azzaro, Divisione di Oculistica Ospedale Civile

Vittoria (Ragusa)

Nunzio Bonanno, Divisione di Oculistica Azienda Ospedaliera Papardo, Messina

Giancarlo Caprioglio, Divisone di Oculistica Ospedale Civile, Venezia

Pasquale D’Arrigo, Divisione di Oculistica Ospedale R Margherita, Messina

Enrico Gandolfo, Clinica Oculistica Università di Brescia Roberto Ratiglia, Clinica Oculistica Università di Milano Alfredo Reibaldi, Clinica Ocuslitica Università di Catania Aldo Scialfa, Divisione di Oculistica Ospedale Garibaldi, Catania

Francesco Zagari, Divisione di Oculistica Azienda Ospedaliera Cannizzaro, Catania 